\title{
SEARCHES FOR R-PARITY VIOLATION PROCESSES IN DELPHI
}

\author{
V. POIREAU \\ CEA Saclay DSM/DAPNIA/SPP \\ 91191 Gif sur Yvette cedex, France \\ E-mail:vincent.poireau@cern.ch
}

\begin{abstract}
We present a review of searches of supersymmetric signal with R-parity violation in DELPHI. An introduction on R-parity is given, followed by a presentation of the different signatures at LEP. The experimental searches are described for each channel and the results are summarized in a final table.
\end{abstract}

\section{Introduction on R-parity violation}

The most general superpotential of the minimal supersymmetric standard model (MSSM) can be expressed in a sum of two terms:

$$
W_{M S S M}=W_{R_{p}}+W_{R_{p}},
$$

where $W_{R_{p}}$ is the classical term of the $\operatorname{MSSM}^{1}$ and where $W_{R_{p}}$ reads $^{a}$ :

$$
W_{\lambda_{p}}=\lambda_{i j k} L_{i} L_{j} E_{k}^{c}+\lambda_{i j k}^{\prime} L_{i} Q_{j} D_{k}^{c}+\lambda_{i j k}^{\prime \prime} U_{i}^{c} D_{j}^{c} D_{k}^{c} \text {. }
$$

The numbers $i, j$ and $k$ are the generation indices; $L$ and $E$ represent respectively the left-handed doublet and the right-handed singlet of the lepton superfield; $Q, U$ and $D$ denote respectively the left-handed doublet, the uptype right-handed singlet and the down-type right-handed singlet of the quark superfields; $\lambda_{i j k}, \lambda_{i j k}^{\prime}$ and $\lambda_{i j k}^{\prime \prime}$ are Yukawa couplings shown on figure 1.
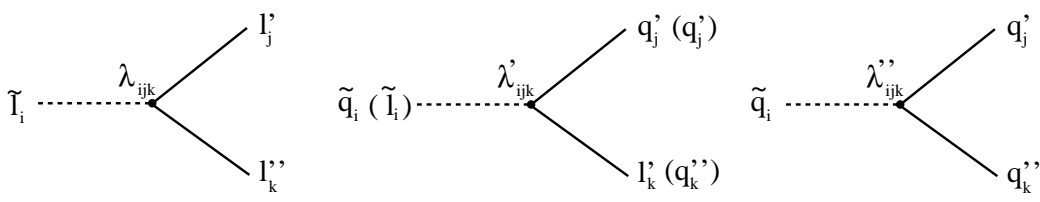

Figure 1. Couplings involved in the $W_{R R_{p}}$ superpotential.

From the superpotential, we observe that $\lambda$ and $\lambda^{\prime}$ couplings violate the lepton number $(L)$ conservation and that $\lambda^{\prime \prime}$ couplings violate the baryon

${ }^{a}$ We made here the assumption that the term $\mu_{i}^{\prime} H_{2} L_{i}$ of the superpotential could be rotated away.

RPV: submitted to World Scientific on July 6, 2001 
number $(B)$ conservation (since a superparticle carries the same quantum numbers as its standard partner, except for the spin). A direct consequence of these violations is a possible decay of the proton (via for example the $\lambda^{\prime}$ and $\lambda^{\prime \prime}$ couplings). In $1975^{2}$, a new discrete symmetry, the R-parity associated to a multiplicative quantum number, $R_{p}$, was introduced in order to suppress the $W_{R_{p}}$ term and consequently to avoid the proton decay. The R-parity was defined as $R_{p}=(-1)^{3 B+L+2 S}$, where $S$ is the spin of the particle. With this definition, a standard particle obeys $R_{p}=1$ and a supersymmetric one obeys $R_{p}=-1$. If we ask for the conservation of R-parity, the coupling constants of figure 1 are suppressed, and only the $W_{R_{p}}$ term of the superpotential remains. This model may be called the MSSM with R-parity conservation.

The introduction of the R-parity was motivated for phenomenological reasons: conservation of $L$ and $B$, and proton stability. However, the proton decay can be avoided if we assume that only one coupling dominates at a time. Furthermore, $L$ and $B$ violation are allowed if their level are below experimental limits. Finally, no strong theoretical reason can be given for the suppression of couplings which appear naturally in the most general superpotential. Thus, we consider here models where we keep the $W_{P_{p}}$ term in the superpotential. This term does not conserve the R-parity, and therefore this model is called MSSM with R-parity violation.

At the phenomenological level, the main consequence is that the lightest supersymmetric particle (LSP) can decay into standard particles and therefore may be visible within the detector volume. Furthermore, one single superparticle can be produced in the collision of two standard particles.

The working hypotheses in this paper are the following. First, we use the framework of the constrained MSSM, which depends on 5 parameters ${ }^{1}: M_{2}$ the $\mathrm{SU}(2)$ gaugino mass at the electroweak scale, $m_{0}$ the sfermion mass at the grand unified scale, $A_{0}$ the trilinear term at the grand unified scale, $\tan \beta$ the ratio of the vacuum expectation values of the two Higgs doublets and $\mu$ the mixing mass term of the Higgs doublets. The LSP is assumed to have a negligible lifetime so that the production and decay vertices coincide. Finally, the assumption is made that only one R-parity violating coupling is dominant at a time.

\section{R-parity violation signatures at LEP}

\subsection{Production}

The pair production of superparticles is exactly similar to the R-parity conserved case. The R-parity violating couplings are only involved in the sub- 
sequent decays of the produced superparticles (see below). Different kinds of pair productions were studied in DELPHI: slepton, squark, neutralino and chargino pair production. In addition, on the contrary of the R-parity conserved case, one superparticle may be produced from a standard particle collision. At LEP, a sneutrino can be singly produced via $\lambda_{121}$ or $\lambda_{131}$ couplings.

\subsection{Decay}

Two kinds of decay of the produced superparticles are possible: the direct and the indirect decays. If the superparticle is the LSP, it will decay into standard particles, possibly via an off-shell superparticle: this is the so-called direct decay. If the superparticle is not the LSP, it will first decay into the LSP and a standard particle (for example a W boson), then the LSP will decay directly into standard particles. The difference of mass between the produced superparticle and the LSP is called $\Delta M$. The final state depends strongly on the dominant coupling constants. For $\lambda$ couplings, the final state is mainly composed of leptons. For $\lambda^{\prime}$ couplings, the final state consists of a mixed state of jets and leptons whereas for $\lambda^{\prime \prime}$ couplings it contains a large number of jets.

\section{Searches in DELPHI}

The DELPHI experiment ${ }^{3}$, located on the LEP ring at CERN, recorded an integrated luminosity of $226 \mathrm{pb}^{-1}$ in 1999 and of $224 \mathrm{pb}^{-1}$ in 2000, with energy ranging from $192 \mathrm{GeV}$ to $208 \mathrm{GeV}$. Although lots of topologies were treated by DELPHI, only $\lambda$ and $\lambda^{\prime \prime}$ R-parity violating ${ }^{b}$ couplings were covered ${ }^{4}$. The results of the analyses are presented in the following sections. Excluded domains in the MSSM parameter space and limits presented below are given at the $95 \%$ confidence level.

\subsection{Charginos and neutralinos}

If we assume that the dominant coupling is $\lambda$, then the final state consists of leptons and missing energy coming from neutrinos, and additional jets or leptons if the superparticle decay is indirect. The nature of leptons depends on the type of the $\lambda$ coupling constant. Two analyses have been performed: one with a $\lambda_{122}$ coupling which involves electrons and muons (most efficient case) and one with a $\lambda_{133}$, which represents the worse case due to the taus

${ }^{b}$ Spontaneous R-parity violating signals were also searched for in DELPHI ${ }^{5}$, but are not described here.

RPV: submitted to World Scientific on July 6, 2001 
in the final state. The analyses were based on missing quantities, lepton identification and kinematic properties, and jet characteristics.

If we assume that the dominant coupling is $\lambda^{\prime \prime}$, the final state contains 6 to 10 jets. These multijet events are present in the standard model via four-fermion processes with gluon radiations. A jet algorithm was used to characterize the signal and to provide powerful variables for the discrimination between signal and background events.

For both analyses, no excess was found in the data with respect to the standard model expectations. These results translate into limits on the parameters $\mu, M_{2}, \tan \beta$ and $m_{0}$ (notice that the cross-section does not depend on the parameter $A_{0}$ ). Figure 2 shows examples of exclusion domains obtained from these analyses for $\tan \beta=1.5$ and $m_{0}=90 \mathrm{GeV} / c^{2}$. From a scanning on the parameters, we obtained limits on the superparticle masses. The chargino mass limit was $103 \mathrm{GeV} / c^{2}$ for dominant $\lambda$ couplings and $102.5 \mathrm{GeV} / c^{2}$ for $\lambda^{\prime \prime}$ couplings. Concerning the neutralino mass, the limits were $40 \mathrm{GeV} / c^{2}$ for $\lambda$ couplings and $38 \mathrm{GeV} / c^{2}$ for $\lambda^{\prime \prime}$ couplings.
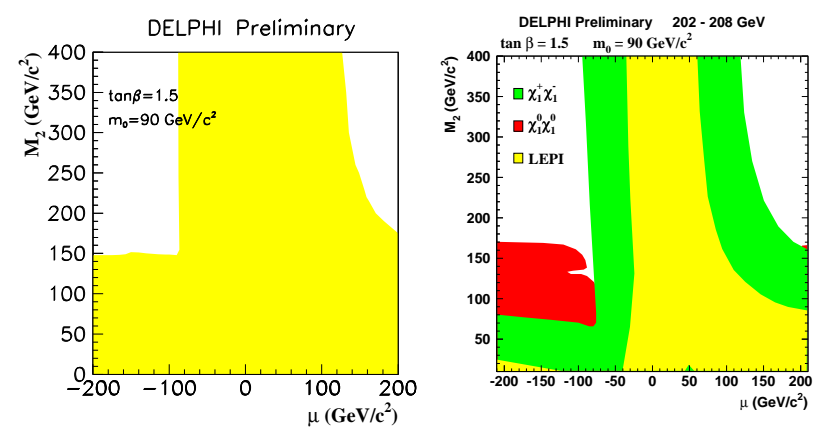

Figure 2. Exclusion plots in the $\left(\mu, M_{2}\right)$ plane for $\tan \beta=1.5$ and $m_{0}=90 \mathrm{GeV} / c^{2}$ (left-hand side: $\lambda$ couplings; right-hand side: $\lambda^{\prime \prime}$ couplings).

\subsection{Sleptons}

In the case of a dominant $\lambda$ coupling, the sneutrino pair production was carefully studied. The direct decay of the two sneutrino gives a 4 lepton final state, whereas the indirect decay gives 4 leptons and missing energy. Furthermore, the indirect decay of charged slepton pair was studied. The final state of this signal is composed of 6 leptons and missing energy. For all these topologies, different analyses were performed depending on the slepton 
flavor and the indices of coupling. No excess of candidate events was found in the 1999 and 2000 data.

In the hypothesis of a $\lambda^{\prime \prime}$ dominance, only the indirect decay is possible via this coupling. The selectron and the smuon were searched for in the data. These pair productions give 2 leptons (electrons or muons) and 6 jets. These analyses combined the use of lepton identification and jet algorithm, resulting in a good rejection power. No excess of data over standard model backgrounds was observed.

These results can be interpreted in the framework of the constrained MSSM. The analyses from 1999 and 2000 data were combined to exclude an area in the plane of the neutralino mass versus the slepton mass. Figure 3 presents examples of exclusion domains, from which limits on slepton masses can also be derived. For the $\lambda$ couplings, a limit of $87 \mathrm{GeV} / c^{2}$ was obtained on the slepton masses (charged sleptons and sneutrinos). Concerning $\lambda^{\prime \prime}$ couplings, limits were $92 \mathrm{GeV} / c^{2}$ for the $\widetilde{e}_{R}$ and $85 \mathrm{GeV} / c^{2}$ for the $\widetilde{\mu}_{R}$.
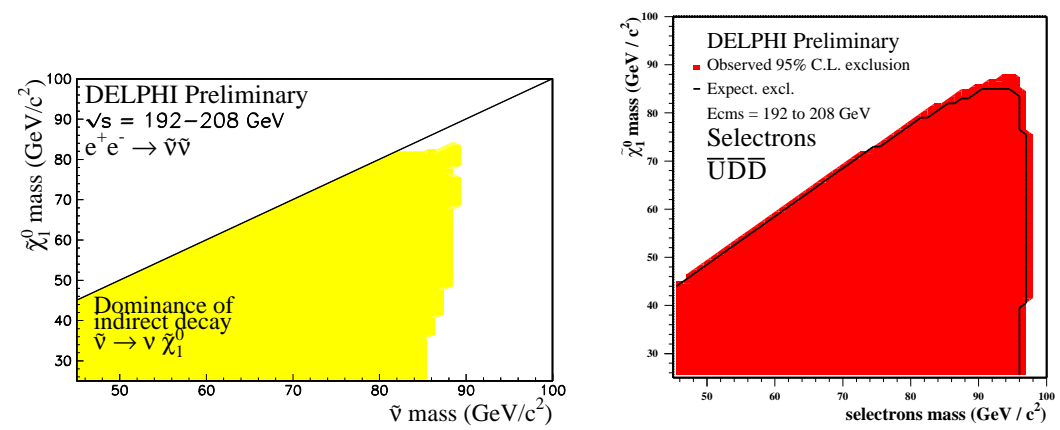

Figure 3. Left: excluded region for sneutrino production for indirect decays ( $\lambda$ couplings). Right: exclusion domain for the indirect selectron decay ( $\lambda^{\prime \prime}$ couplings).

\subsection{Squarks}

The mixing between right- and left-handed stops can be important, so that the mixed state $\widetilde{t}_{1}$ is expected to be the lightest squark. Each of the produced stop decays into a charm quark and a neutralino, giving a signature with jets, charged leptons and neutrinos in the case of $\lambda$ dominance, and a signature with 8 jets in the case of dominant $\lambda^{\prime \prime}$ couplings. For both couplings, agreement between data and standard model expectations was satisfactory. Thus, exclusion regions were derived from these results, with the conservative hy- 
pothesis of maximal decoupling to the $\mathrm{Z}$ boson (figure 4 ). Limit on $\widetilde{t}_{1}$ mass was found to be $87 \mathrm{GeV} / c^{2}$ for $\lambda$ and $75 \mathrm{GeV} / c^{2}$ for $\lambda^{\prime \prime}$ couplings (in the case of maximal Z-decoupling).
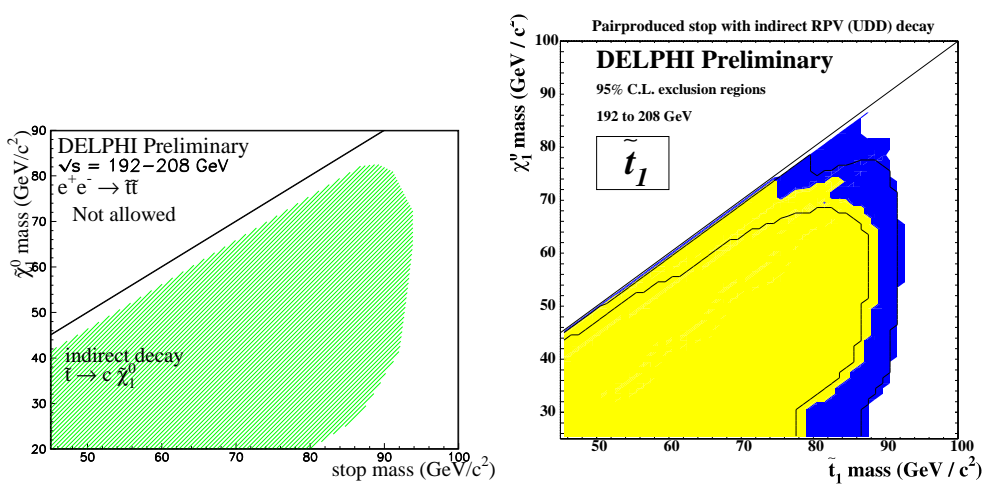

Figure 4. Left: exclusion domain for the $\widetilde{t}_{1}$ production for $\lambda$ couplings (maximal decoupling). Right: exclusion domain for the $\widetilde{t}_{1}$ production for $\lambda^{\prime \prime}$ couplings (light grey: maximal decoupling; dark grey: no mixing).

\subsection{Sneutrino single production}

A resonant single production is allowed at LEP via the $\lambda_{121}$ or $\lambda_{131}$ coupling constants. The cross-section of the process depends directly on these couplings, so that searches are sensitive to their values. The decay channels $\widetilde{\nu} \longrightarrow \widetilde{\chi}^{0} \nu$ and $\widetilde{\nu} \longrightarrow \widetilde{\chi}^{ \pm} l^{\mp}$ were searched for in the data (only for the $\lambda_{121}$ coupling). Depending on the decay of the neutralino and chargino, the final state is either purely leptonic or semi-leptonic (with additional jets). After a complete analysis, the number of observed events was in agreement with the number of expected events. This result allowed to derive limits on the $\lambda_{121}$ coupling: figure 5 shows this exclusion for $\tan \beta=1.5$ (with the hypothesis that $\left.\Gamma_{\tilde{\nu}} \geq 0.1 \mathrm{GeV} / c^{2}\right)$.

\section{Conclusions}

R-parity violating signatures have been studied in the DELPHI detector: a large number of topologies was covered. No excess was observed with respect to the standard model predictions, which was interpreted to constrain the 


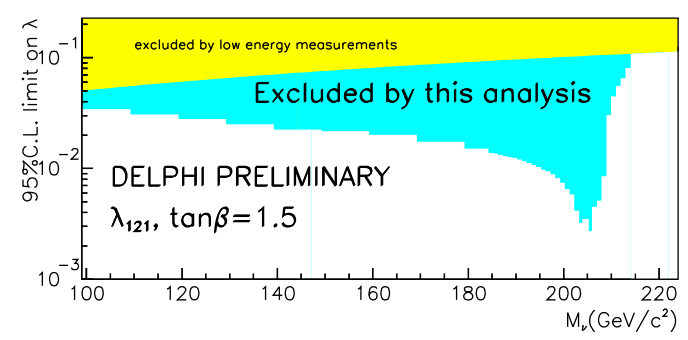

Figure 5. Upper limit on $\lambda_{121}$ as a function of the sneutrino mass.

MSSM parameter space. The mass limits derived from these searches are summarized in table 1 for different R-parity violating couplings.

Table 1. Superparticle mass limits in $\mathrm{GeV} / c^{2}$ from the DELPHI searches.

\begin{tabular}{|c|c|c|}
\hline Superparticle & $\lambda$ & $\lambda^{\prime \prime}$ \\
\hline \hline$\widetilde{\chi}_{1}^{0}$ & $\mathbf{4 0}$ & $\mathbf{3 8}$ \\
$\widetilde{\chi}_{1}^{ \pm}$ & $\mathbf{1 0 3}$ & $\mathbf{1 0 2 . 5}$ \\
\hline$\widetilde{e}_{R}$ & $\mathbf{8 7}^{1,2}$ & $\mathbf{9 2}$ \\
$\widetilde{\mu}_{R}$ & $\mathbf{8 7}^{1,2}$ & $\mathbf{8 5}^{1,3}$ \\
$\widetilde{\tau}_{R}$ & $\mathbf{8 7}^{1,2}$ & - \\
\hline
\end{tabular}

\begin{tabular}{|c|c|c|}
\hline Superparticle & $\lambda$ & $\lambda^{\prime \prime}$ \\
\hline \hline$\widetilde{\nu}_{e}$ & $\mathbf{8 7}^{1}$ & - \\
$\widetilde{\nu}_{\mu}$ & $\mathbf{8 7}^{1}$ & - \\
$\widetilde{\nu}_{\tau}$ & $\mathbf{8 7}^{1}$ & - \\
\hline$\widetilde{t}_{1}$ & $\mathbf{8 7}^{1,3}$ & $\mathbf{7 5}^{1,3}$ \\
\hline
\end{tabular}

1 Obtained for $\mu=-200 \mathrm{GeV} / c^{2}$ and $\tan \beta=1.5$.

2 This result is valid for $\Delta \mathrm{M}>3 \mathrm{GeV} / c^{2}$.

3 This result is valid for $\Delta \mathrm{M}>5 \mathrm{GeV} / c^{2}$.

\section{References}

1. M. Drees, An introduction to supersymmetry, hep-ph/9611409.

2. P. Fayet, Nucl. Phys. B 90, 104 (1975).

3. DELPHI collaboration, Nucl. Instrum. Methods A378, 57 (1996).

4. R. Barbier, C. Bérat, P. Jonsson and V. Poireau, DELPHI 2001-083 CONF 511;

F. Ledroit-Guillon, DELPHI 2001-019 CONF 460.

5. D. Moraes, L. de Paula and M. Gandelman, DELPHI 2001-084 CONF 512. 